\title{
LOCATION OF APPROXIMATIONS OF A MARKOFF THEOREM
}

\author{
K.C. PRASAD \\ Department of Mathematics \\ Ranchi University \\ Ranchi - 834008, India \\ M. LARI \\ Birsa Agriculture University \\ P. SINGH \\ S.S.M. College \\ Ranchi, India \\ (Received November 26, 1986)
}

ABSTRACT. Relative to the first two theorems of the well known Markoff Chain (J.W.S. Cassels, "An introduction to diophantine approximation" approximations are well located. Literature is silent on the question of location of approximations in reference to the other theorems of the Chain. Here we settle it for the third theorem of the Chain.

KEY WORDS AND PHRASES. Continued fractions, rational approximation.

1980 AMS SUBJECT CLASSIFICATION CODE. 10F05

1. INTRODUCTION

Suppose $\theta$ is an irrational number whose simple continued fraction expansion is $\left[a_{0}, a_{1}, a_{2}, \ldots ., a_{n}, \ldots .\right.$. Let $\alpha_{n}(\theta)$ denote

$$
\left[0, a_{n}, a_{n-1}, \ldots, a_{1}\right]+\left[a_{n+1}, a_{n+2}, \ldots\right]
$$

Markoff Chain (Cassels [1], Kokshma [2]) is the following chain of theorems about the sequence $\left\{\alpha_{n}(\theta)\right\}_{n} \geqq 1$ :

$\mathrm{T}_{1}$ : For every irrational number $\theta$,

$$
\alpha_{j}(\theta)>\sqrt{5}
$$

for infinity of $j^{\prime} s$ and $\sqrt{5}$ cannot be increased for $\theta \sim\left[0,(1)^{*}\right]$.

$\mathrm{T}_{2}:$ If $\theta+\left[0,(1)^{*}\right]$, then

$$
\alpha_{j}(\theta)>\sqrt{8}
$$

for infinity of $j^{\prime} s$ and $\sqrt{8}$ cannot be increased for $\theta \sim\left[0,(2)^{*}\right]$. 
$\mathrm{T}_{3}:$ If $\theta+\left[0,(1)^{*}\right]$ or $\left[0,(2)^{*}\right]$ then

$$
\alpha_{j}(\theta)>(\sqrt{221}) / 5
$$

for infinity of $j^{\prime} s$ and $(\sqrt{221}) / 5$ cannot be increased for $\theta \sim\left[0,(2,2,1,1)^{*}\right]$

$\mathrm{T}_{4}$ : If $\theta+\left[0,(1)^{*}\right]$ or $\left[0,(2)^{*}\right]$ or $\left[0,(2,2,1,1)^{*}\right]$ then

$$
\alpha_{j}(\theta)>(\sqrt{1517}) / 13
$$

for infinity and $j^{\prime} s$ and $(\sqrt{1517}) / 13$ cannot be increased for

$$
\theta+\left[0,(2,2,1,1,1,1)^{*}\right] \text { etc. }
$$

It is know that the sequence of constants $\sqrt{5}, \sqrt{8},(\sqrt{221}) / 5,(\sqrt{1517}) / 13, \ldots$, increases to 3 . So the theorems say something non-trivial about $\theta^{\prime} s$ in which all quotients are eventually 1 or 2 only.

As regards (1.1) and (1.2) we have an ad-hoc idea of the $j$ 's satisfying them. In reference to $T_{1}$ we know that : one $j$ must occur in $\{n, n+1, n+2\} \forall n \geqq 1$. Relative to $T_{2}$, we have a similar result : if $a_{n+2}=2$ then a $j \varepsilon\{n, n+1, n+2\}$. These may be found in Wright [3] or Prasad and Lari [4].

But the literature is surprisingly silent on such results in reference to $\mathrm{T}_{3}, \mathrm{~T}_{4}$, etc. In this article we announce one such result in reference to $T_{3}$ in the following theorem:

2. MAIN RESULTS

THEOREM. $a_{n+2}=2$ and $a_{n+3}=1$

then $\alpha_{j}(\theta)>(\sqrt{221}) / 5$ for at least one $j \varepsilon\{n, n+1, n+4\}$

REMARK. Our method gives a way to try for similar results on $\mathrm{T}_{4}, \mathrm{~T}_{5}$, etc.

PROOF. Suppose $\theta=\left[a_{0}, a_{1}, \ldots, a_{n}, \ldots\right], a_{n+2}=2$ and $a_{n+3}=1$

If $a_{n+1} \geqq 3 ; \alpha_{n}(\theta)>3$ and we are through.

If $a_{n+1}=1 ; a_{n+1}(\theta)>[0,2]+[2,2]=3$ and we are through.

If $a_{n+1}=2$ and $a_{n+4} \geq 2$ then $a_{n+1}(\theta)>[0,3]+[2,1,1]=3$ and we are through.

For the left out $\theta^{\prime} s$ : $a_{n+1}=2=a_{n+2}$ and $a_{n+3}=1=a_{n+4}$.

To deal with them, we put

$$
\begin{aligned}
& \alpha=\left[0, a_{n}, a_{n-1}, \ldots, a_{1}\right], \\
& \beta=\left[0, a_{n+5}, a_{n+6}, \ldots . .\right], \\
& t=(\sqrt{221}) / 5
\end{aligned}
$$

and argue over all possible values of $\beta$. We note:

$$
\begin{aligned}
& { }^{\prime} \alpha_{n+4}(\theta)>t^{\prime} \Leftrightarrow{ }^{\prime} \alpha[5-(5 t-3) \beta]>[(2 t-7) \beta-12]^{\prime} \\
& \text { So } \beta \leqq \frac{12}{12 t-7} \Rightarrow \alpha_{n+4}(\theta)>t
\end{aligned}
$$


We next check:

$$
\begin{aligned}
& \alpha_{n}(\theta)>t \Leftrightarrow \alpha>f_{1}(\beta)=\frac{(5 t-12)+(3 t-7) \beta}{(5+3 \beta)} \\
& \alpha_{n+1}(\theta)>t \leftrightarrow \alpha<f_{2}(\beta)=\frac{(12-4 t)+(7-2 t) \beta}{(2 t-5)-(3-t) \beta} \\
& \text { and } f_{2}(\beta)-f_{1}(\beta)=A_{1}\left(\beta+\frac{251-5 t}{146}\right)\left(\beta-\frac{5 t-9}{14}\right) \\
& \text { where } A_{1}=t(10-3 t)(5+3 \beta)^{-1}\{(2 t-5)-(3-t) \beta\}^{-1}:(>0) \text {. } \\
& \text { So } \beta>\frac{5 t-9}{14} \\
& \Rightarrow \mathbf{f}_{2}(\beta)>\mathbf{f}_{1}(\beta) \\
& \Rightarrow \alpha>f_{1}(\beta) \text { or } \alpha<f_{2}(\beta) \\
& \Rightarrow \quad \alpha_{n}(\theta)>t \text { or } \alpha_{n+1}(\theta)>t \text {. }
\end{aligned}
$$

Hence we confine attention to $\frac{12}{12 t-7}<\beta \leqq \frac{5 t-9}{14}$

In this case $\alpha_{n+4}(\theta)>t \Leftrightarrow \alpha>f_{3}(\beta)=\frac{(12 t-7) \beta-12}{5-(5 t-3) \beta}$

Also $f_{3}(\beta)-f_{2}(\beta)=A_{2}\left(\beta+\frac{5 t+19}{14}\right)\left(\beta-\frac{5 t-9}{14}\right)$

where $A_{2}=2 t(t-1)\{5-(5 t-3) \beta\}^{-1} \cdot\{(2 t-5)-(3-t) \beta\}^{-1}:(>0)$

So if $\frac{12}{12 t-7}<\beta<\frac{5 t-9}{14}$ then $\mathrm{f}_{3}(\beta)<\mathrm{f}_{2}(\beta)$

which implies $\alpha<f_{2}(\beta)$ or $\alpha>f_{3}(\beta)$; equivalently

$\alpha_{n+1}(\theta)>t$ or $\alpha_{n+4}(\theta)>t$ and we are through.

Finally $\beta=\frac{5 t-9}{14}$

$\Rightarrow f_{1}(\beta)=f_{2}(\beta)=f_{3}(\beta)=\frac{5 t-9}{10} \quad$ (an irrational number)

$\Rightarrow \alpha>\mathbf{f}_{1}(\beta)$ or $\alpha<\mathbf{f}_{2}(\beta), \quad(\alpha$ is rational)

$\Rightarrow \alpha_{n}(\theta)>t$ or $\alpha_{n+1}(\theta)>t$.

This completes the proof of the theorem. 


\section{REFERENCES}

1. CASSELS, J.W.S. An Introduction to Diophantine Approximation, Cambridge Tracts No. 45, Cambridge, 1957.

2. KOKSMA, J.F. Diphatische Approximation, Chelsea, New York, 1936.

3. WRIGHT, E.M. Approximation of Irrationals by Rationals, Math. Gaz. 48 (1964), 288-89.

4. PRASAD, K.C. and LARI, M. A Note on a Theorem of Perron; Proc. Amer. Math. Soc. 97 (1986) 19-20. 


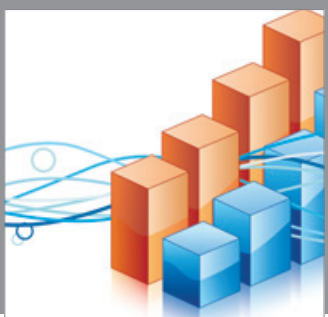

Advances in

Operations Research

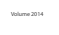

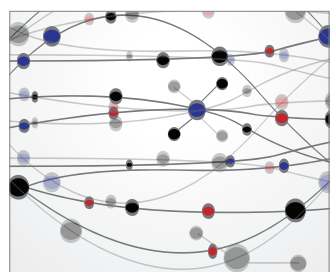

\section{The Scientific} World Journal
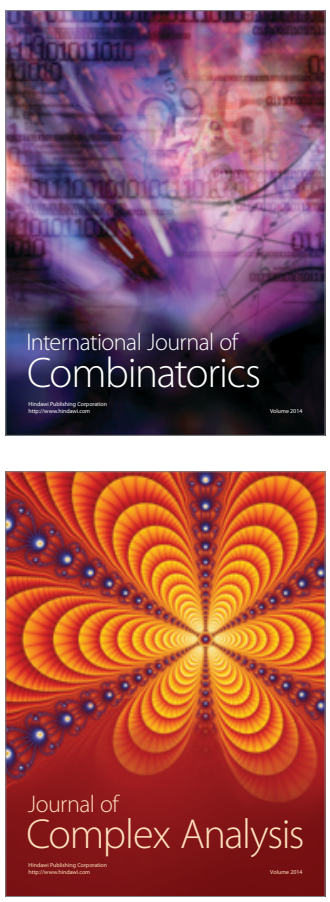

International Journal of

Mathematics and

Mathematical

Sciences
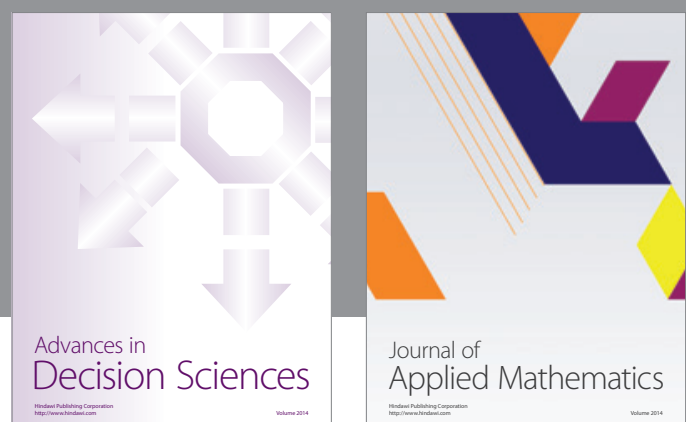

Journal of

Applied Mathematics
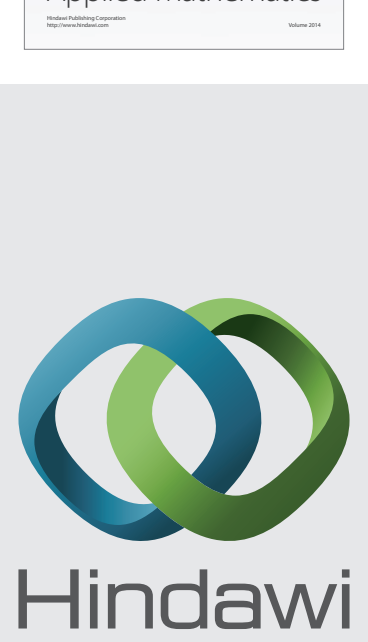

Submit your manuscripts at http://www.hindawi.com
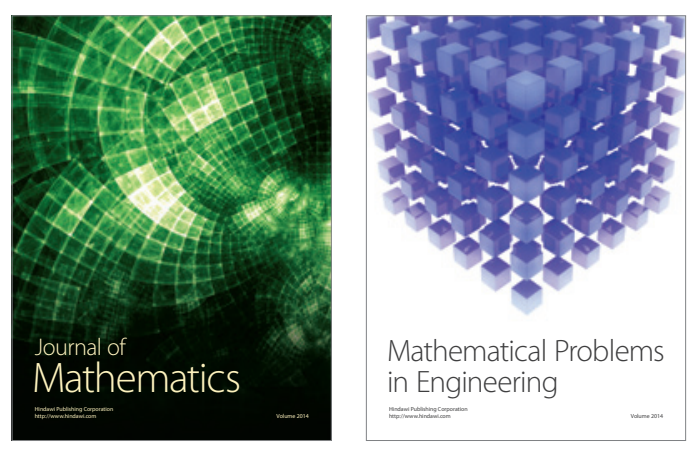

Mathematical Problems in Engineering
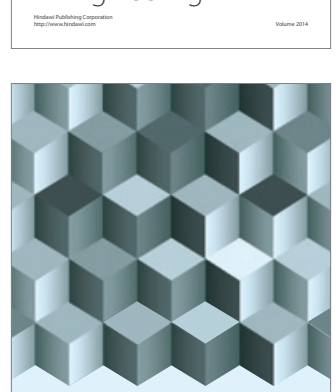

Journal of

Function Spaces
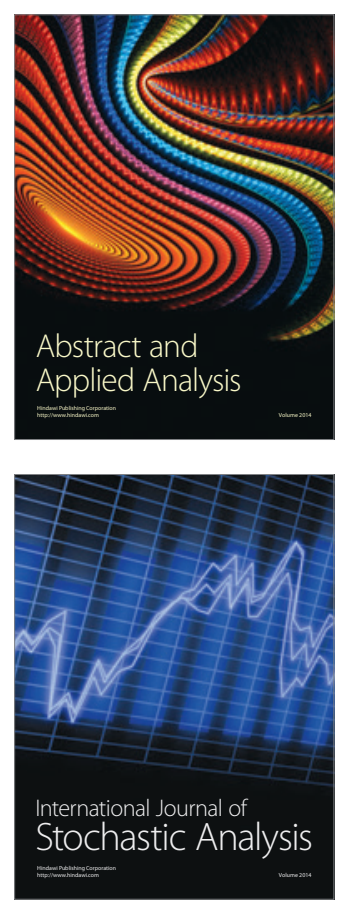

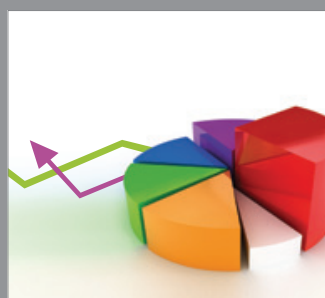

ournal of

Probability and Statistics

Promensencen
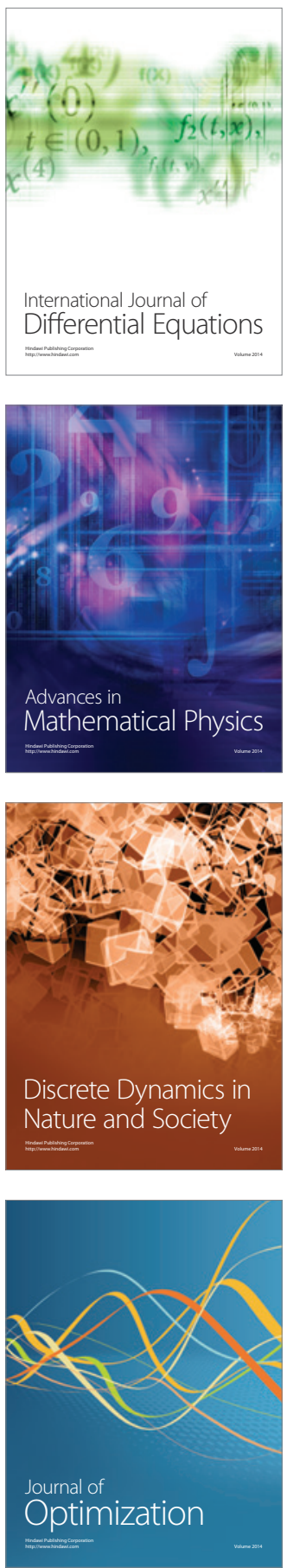